\title{
BRAESS'S PARADOX AND POWER-LAW NONLINEARITIES IN NETWORKS
}

\author{
BRUCE CALVERT ${ }^{1}$ and GRANT KEADY ${ }^{2}$
}

(Received 12 July 1991; revised 5 December 1991)

\begin{abstract}
We study flows in physical networks with a potential function defined over the nodes and a flow defined over the arcs. The networks have the further property that the flow on an $\operatorname{arc} a$ is a given increasing function of the difference in potential between its initial and terminal node. An example is the equilibrium flow in water-supply pipe networks where the potential is the head and the Hazen-Williams rule gives the flow as a numerical factor $k_{a}$ times the head difference to a power $s>0$ (and $s \cong 0.54$ ). In the pipe-network problem with Hazen-Williams nonlinearities, having the same $s>0$ on each arc, given the consumptions and supplies, the power usage is a decreasing function of the conductivity factors $k_{a}$. There is also a converse to this. Approximately stated, it is: if every relationship between flow and head difference is not a power law, with the same $s$ on each arc, given at least 6 pipes, one can arrange (lengths of) them so that Braess's paradox occurs; i.e. one can increase the conductivity of an individual pipe yet require more power to maintain the same consumptions.
\end{abstract}

\section{Introduction}

Braess in [2] gave an example of a network, in the setting of traffic flow, in which an extra arc (i.e. road) was added and the total travel time for any road user was increased. Braess's example has just one origin-destination pair, so that it is, like the physical network flows in this paper, a single-commodity

\footnotetext{
'Mathematics Department, University of Auckland, New Zealand

${ }^{2}$ Mathematics Department, University of Western Australia, Nedlands 6009

(C) Australian Mathematical Society, 1993, Serial-fee code 0334-2700/93
} 
flow. (See Rockafellar [21, Section 1K], for definitions.) The phenomenon was reconsidered in several works on traffic flow (such as Murchland [18], LeBlanc [16], Fisk [14]) and in popularisations (such as Toint [23]).

Nonlinear networks occur in many contexts, see Dembo et al. [10], Rockafellar [21]. In the context of flows in physical networks, Braess's paradox can be restated informally as follows.

BRAESS'S PARADOX. The power consumed in a nonlinear network can increase if an arc's conductivity is increased with consumptions held constant.

Theorem 11 of the present paper states that Braess's paradox cannot occur in a two-terminal series-parallel network. See Keady [15, Part I, Appendix A], for a discussion.

One of the examples of physical networks is the flow of water in a pipe network. When the commonly-used Hazen-Williams rule (with the same exponent on each arc, e.g. Brebbia and Ferrante [3]) is assumed, our Theorem 1 shows that Braess's paradox cannot occur. This was known already in two-terminal resistive networks where Ohm's law holds for each resistor, that is, $s=1$ for all $\operatorname{arcs} a$, as shown in Shannon and Hagelbarger [22], Melvin [17].

Define a network to be of type $(\mathrm{H})$ if, for each $\operatorname{arc} A$, the flow is a numerical factor $k_{a}$ times the head difference to a power $s>0$ independent of $a$. (The definition of type $(\mathrm{H})$ has been introduced as an aid to readers because there are two separate meanings of the word "power" in this paper.) We provide various characterisations, both of when a network is type $(\mathrm{H})$, and also of when a network can exhibit Braess's paradox. The two subjects are related: see our main theorems, Theorems 1 and 2 of Section 3. In a recent paper on Braess's paradox, Cohen and Horowitz [8] write: "The task remains of specifying the general conditions under which such paradoxes can occur, for general network topologies and broad classes of components ...". Our theorems summarise some progress with this task.

Our first exposition, in [15], treated these matters in the setting of twoterminal networks. Rockafellar [21, Section 8N], calls these "black boxes". Here the exposition is in terms of the power-loss in a general network, with known consumptions at each node. Two-terminal networks are just a special case. 


\section{Notation}

Our notation follows that of Bertsekas et al. [1] and of Rockafellar [21]. A network $G=(N, A)$, or the directed graph associated with the network, consists of two finite sets $A$ and $N$ and a function that assigns to each $a \in A$ a pair $(i, k) \in N \times N$ such that $i \neq k$. The elements of $A$ are called arcs (or edges); the elements of $N$ are called nodes (or vertices). We may label the arcs with the first $|A|$ positive integers, i.e. starting from 1 , and identify $A$ with this set. We label the nodes with the $|N|$ nonnegative integers from 0 to $|N|-1$. The correspondence of $\operatorname{arc} j$ with its nodes is written $j \sim(i, k)$. We say that $i$ is the initial or start node of arc $j$ and $k$ is the terminal or end node of arc $j$.

Let $E$ be a node-arc incidence matrix for $G$; that is, assign a column vector to each arc, with 0 everywhere except for a 1 for one node of the arc and -1 for the other. More precisely, the entries $e_{i j}$ of $E$ are given by

$$
e_{i j}= \begin{cases}+1 & \text { if } i \text { is the initial node of } \operatorname{arc} j \\ -1 & \text { if } i \text { is the terminal node of } \operatorname{arc} j \\ 0 & \text { otherwise }\end{cases}
$$

We always assume that $G$ is connected, so that $|A| \geq|N|-1$, and the rank of $E$ is $|N|-1$.

For $i \in N$, we let $p(i) \in \mathbb{R}$ be the head (or potential or voltage or time) at node $i$. For $a \in A$, let $q_{a} \in \mathbb{R}$ denote the flow on $\operatorname{arc} a$, from start to end. For $i \in N$, let $b(i)$ be the deficit (or input, or consumption, or current supplied) at node $i$.

For each arc $a \sim(i, k) \in A$, suppose there is given a conductivity function $\sigma_{a} \mathbb{R} \rightarrow \mathbb{R}$ and a nonnegative real number, the conductivity factor $k_{a}$. The flow function $k_{a} \sigma_{a}$ will relate the head differences and flows by (2.2).

DEFINITION. We say the network conductivity functions satisfy Assumption $A(\sigma)$ if, for each $a \in A, \sigma_{a}$ is continuous, $\sigma_{a}(0)=0, \sigma_{a}(-t)=-\sigma_{a}(t)$ for all $t, \sigma_{a}(t) \rightarrow \infty$ as $t \rightarrow \infty, \sigma_{a}$ is $C^{\prime}$ on $(0, \infty)$, and $\sigma_{a}^{\prime}(t)>0$ for all $t>0$.

DEFINITION. We say the network conductivity factors satisfy Assumption $A(k)$ if, with $A(k)=\left\{a \in A \mid k_{a}>0\right\},(N, A(k))$ is a connected graph.

As all of our results depend on $G$ being connected and assumption $A(k)$ being satisfied, these are presumed to be satisfied throughout the paper. 
Rockafellar [21, Section $8 \mathrm{H}$ ], defines the network equilibrium problem as follows. Given $b$ with

$$
\sum_{i \in N} b(i)=0,
$$

given conductivity functions $\sigma_{a}$ satisfying Assumption $A(\sigma)$, and given conductivity factors $k_{a}$ satisfying Assumption $A(k)$, find a head vector $p \in \mathbb{R}^{|N|}$ such that

$$
q_{a}=k_{a} \sigma_{a}(p(i)-p(k)), \quad \forall a \sim(i, k) \in A,
$$

and

$$
E q=b .
$$

We remark that the above only determines $p$ up to a $p+c e, c \in \mathbb{R}$, where $e$ is the vector all of whose entries are one. When, as from Section 4 onwards, we want to make $p$ unique, we suppose its 0th entry is 0 .

Informally, the $\sigma_{a}$ can be thought of as giving the form of the conductivity law. In the context of the pipe-network problem with Hazen-Williams flow functions, varying a conductivity factor $k_{a}$ amounts to varying some aspect of pipe $a$ 's geometry, its length or diameter or roughness. Outside the setting of Hazen-Williams laws, varying a conductivity factor seems to be physically unnatural. See the discussion at the end of Section 7.

The "power-law nonlinearity" in our title refers to the situation when the flow $q_{a}$ on an $\operatorname{arc} a \sim(i, k)$ satisfies

$$
q_{a}=k_{a} \sigma\left(p(i)-p(k), s_{a}\right) \quad \text { where } \sigma(t, s)=t|t|^{s-1}, s>0 .
$$

Type $(\mathrm{H})$ means that there exists $s>0$ such that for all $a \in A, \sigma_{a}(t)=\sigma(t, s)$.

The power-loss $P$ in the networks is defined by

$$
P=\sum_{(i, k) \sim a \in A}(p(i)-p(k)) q_{a} .
$$

Where there are arcs in parallel joining $i$ to $k$, the summation is over all of them. We always have $i<k$. Both of these conventions will be used elsewhere in this paper. Provided all the $\sigma_{a}$ are odd functions, the power-loss $\boldsymbol{P}$ is nonnegative and, by Rockafellar [21, Section 11],

$$
P=b^{\top} p .
$$

In a two-terminal network, where $b(i)=0$ except for the two nodes, one of which we always label $i=0$ and the other $n_{t} \leq|N|-1$, we then have 
$P=b(0)\left(p(0)-p\left(n_{t}\right)\right)$. From this, in the language of DC electric circuits, with $b(0)>0$ the applied voltage difference $p(0)-p\left(n_{t}\right)$ is a nonincreasing function of any conductivity factor $k_{a}$ precisely when $P$ is.

Rockafellar [21, Section 8B] gives the following definition.

DEFINITION. The set of pairs $\left(b(0), p(0)-p\left(n_{t}\right)\right)$ when $p$ solves the equilibrium problem is called the characteristic curve of the two-terminal network with terminals 0 and $n_{t}$.

Lemma 3, a variant of Theorem 2, stated in Section 3, implies that, unless the network is type $(\mathrm{H})$, we can increase resistance, or characteristic curve, on an arc, yet lower the overall characteristic curve of the network. This is a restatement, for two-terminal networks, of Braess's paradox.

\section{The main theorems}

THEOREM 1. Suppose $(N, A)$ is a type $(\mathrm{H})$ network and that b satisfies (2.1). Then, for any $a$, the power-loss $P$ is a nonincreasing function of $k_{a}$.

The proof will be given in Section 5 together with a much stronger result with the same hypotheses. Before we state a converse in Theorem 2, we give an additional example of Braess's paradox, this time involving only power-law nonlinearities on the arcs, which shows that it is necessary in Theorem 1 that the powers be the same on all the arcs.

DEFINITION. The Wheatstone bridge graph is the graph $G=(N, A)$ with

$$
N=\{0,1,2,3\}, \quad A=\{(0,1),(0,2),(1,2),(1,3),(2,3)\} .
$$

Four of the arcs form a quadrilateral, the other forms a diagonal.

EXAMPLE OF BRAESS'S PARADOX. Consider the Wheatstone bridge graph, with the diagonal arc $(1,2)$ having variable conductivity. The flow functions $k_{i, j} \sigma_{i, j}$ on the $\operatorname{arcs}(i, j)$ are

$$
k_{01} \sigma_{01}(x)=k_{23} \sigma_{23}(x)=x, \quad k_{02} \sigma_{02}(x)=k_{13} \sigma_{13}(x)=x|x|^{-1 / 2},
$$

and the variable conductivity arc has

$$
k_{12} \sigma_{12}(x)=k x
$$


Choose $b$ such that $b(1)=0=b(2)$, so that the network is a two-terminal one.

The result is that the power-loss $P=b(3)(p(3)-p(0))$ can either decrease or increase as $k$ increases, depending on the value of $b(3)=-b(0)$ and the value of $k$ at the start.

There is a lot of symmetry in the network and the flows, heads and power-loss can be calculated explicitly. The elementary calculations are in [15].

For networks with $|A| \leq 5$, the Wheatstone network is the only two-terminal instance where Braess's paradox can occur. For $|A|>5$, we have the following.

THEOREM 2. Suppose $\left\{\sigma_{a} \mid a \in \mathbb{N}_{\nu}\right\}$ is a set of $v$ functions, $v \geq 6$, satisfying Assumption $A(\sigma)$. Let $\mathcal{N}$ denote the set of all networks $(N, A)$ with flow functions $k_{a} \sigma_{\varphi(a)}$ on arcs $a \in A$, where $\varphi$ ranges over all the one-to-one mappings from $A$ to $\mathbb{N}_{\nu}$. Suppose that for any network $(N, A)$ in $\mathcal{N}$, and any $b$ satisfying (2.1), the power-loss $P$ is a nonincreasing function of each $k_{a}$. Then there is an $s>0$ such that for all $a \in \mathbb{N}_{v}$ and $t \geq 0, \sigma_{a}(t)=\sigma_{a}(1) t^{s}$.

Theorem 2 follows immediately from the following result.

LEMMA 3. Let $\mathcal{N}_{2}$ denote the set of all two-terminal networks $(N, A)$, with the terminal nodes being 0 and $n_{t} \leq|N|-1$. Let $v \geq 6$ and let Assumption $A(\sigma)$ be satisfied as in Theorem 2 . With, in Theorem $2, \mathcal{N}$ replaced by $\mathcal{N}_{2}$ and $b$ satisfying $b(i)=0$ for $i \neq 0, n_{t}$ and $b\left(n_{t}\right)=-b(0)$, the conclusion of Theorem 2 holds. That is, if for any network $(N, A)$ in $\mathcal{N}_{2}$ and any $b$ satisfying the preceding restriction the power-loss $P$ is a nonincreasing function of each $k_{a}$, then there is an $s>0$ such that for all $a \in \mathbb{N}_{\nu}$ and $t \geq 0, \sigma_{a}(t)=\sigma_{a}(1) t^{s}$.

We remark that Theorem 2 and Lemma 3 can be shown to follow from their $v=6$ versions.

The proof of Lemma 3, given in Section 7, depends in part on a detailed analysis of a general nonlinear Wheatstone bridge network.

(If readers find the requirement $v \geq 6$ to be unpleasant, we remark that Theorem 2 can be modified to allow its removal to $v \geq 1$. The modification is to allow repetition of the conductivity functions in the elements in the test networks, i.e. to drop the requirement that $\varphi$ be one-to-one.) 


\section{The convex optimisation problem: primal form}

The pipe-network problem has been treated as an optimisation problem in Duffin [11, 12], Collins et al. [9], Rockafellar [20, 21], Dembo et al. [10].

The proof of Theorem 1 depends on a variational, or optimisation, argument. In Section 2 we defined the network equilibrium problem. Rockafellar [21, Section $8 \mathrm{H}]$ is the standard reference that this problem is equivalent to two others, namely the optimal differential problem, Problem (P) given in the next paragraph, and the optimal distribution problem, Problem (D) given in Section 5. This section contains more material than is needed for the narrow aim of Theorem 1. Some of this, such as existence and uniqueness, is, however, reassuring in that it shows that the counterintuitive flows of Theorem 2 actually exist. The results in this section are not new and, for reasons of space the proofs are suppressed. For detailed proofs and references see [15, 21].

The optimal differential problem defined in Rockafellar [21, Section 8G] is similar to the following. Define

$$
S_{a}(t)=\int_{0}^{t} \sigma_{a}(\hat{t}) d \hat{t}
$$

Define $V_{b}$ by

$$
\begin{aligned}
& V_{0}=\sum_{(i, k) \sim a \in A} k_{a} S_{a}(p(i)-p(k)), \\
& V_{b}=V_{0}-\sum_{i=1}^{|N|-1} b(i) p(i)
\end{aligned}
$$

Define $X$ to be the set of vectors with coordinate indexing starting at 1 in $\mathbb{R}^{|N|-1}$, augmented with a zeroth component which is zero. Problem $(\mathrm{P})$ is to find $p_{*}$ satisfying

$$
V_{b}\left(p_{*}\right)=\min _{p \in X} V_{b}(p)
$$

THEOREM 4. With Assumptions $A(\sigma)$ on the $\sigma_{a}$ and $A(k)$ on the $k_{a}$,

(i) $V_{0}$ is strictly convex on $X$;

(ii) $V_{0}(p) /\|p\| \rightarrow \infty$ as $\|p\| \rightarrow \infty$ in any norm on $X$;

(iii) solutions to Problem $(P)$ exist and are unique in $X$;

(iv) $p_{*}$ solves the network equilibrium problem if and only if it solves Problem (P).

We shall call items (iii)-(iv) Duffin's Existence Theorem. 
An additional property of $V_{0}$, whose consequences are explained in [15] but which is not used in this paper, is the following. For $\sigma_{a}$ satisfying Assumption $A(\sigma)$ in the pipe-network problem, we have the inequality

$$
V_{0}\left(p_{0} \wedge p_{1}\right)+V_{0}\left(p_{0} \vee p_{1}\right) \leq V_{0}\left(p_{0}\right)+V_{0}\left(p_{1}\right) \quad \forall p_{0}, p_{1}
$$

Here $p_{0} \wedge p_{1}$ denotes the minimum of the two vectors $p_{0}, p_{1}$ and $p_{0} \vee p_{1}$ denotes their maximum.

In the case of the Hazen-Williams $\sigma$ we have the following additional item.

THEOREM 5. For type $(\mathrm{H})$ networks, $\left(V_{0}(p)\right)^{1 /(s+1)}$ is a strictly convex norm on $X$.

\section{Duality}

For $\sigma_{a}$ satisfying Assumption $A(\sigma), \sigma_{a}$ has an inverse. Call the inverse function $\rho_{a}$,

$$
\rho_{a}\left(\sigma_{a}(t)\right)=t, \quad \forall t \in \mathbb{R} .
$$

Define $R$ to be the integral of $\rho$ from $0, R(0)=0$. For $q \in \mathbb{R}^{|A|}$ define

$$
U(q)=\sum_{a \in A} k_{a} R_{a}\left(\frac{q_{a}}{k_{a}}\right) .
$$

The problem of minimising $U(q)$ over $q$ satisfying

$$
E q=b
$$

is Problem (D). Recall that $e^{\top} b=0$, where $e$ denotes the vector all of whose entries are 1. Problem (D) is a convex separable programming problem, which Rockafellar [21, Section 8D] calls the optimal distribution problem.

(The functions $R_{a}$ and $S_{a}$ are Fenchel conjugate convex functions on $\mathbb{R}$. For $\sigma_{a}$ a power law, both $R_{a}$ and $S_{a}$ are also power laws.)

Collins et al. [9] establish the following theorem. See also Rockafellar [21, Section 8L]. The notation involving the $\sigma \mathbb{R}^{|A|} \rightarrow \mathbb{R}^{|A|}$, i.e. acting on vectors, is that $\sigma(q)$ is the vector with components $\sigma_{a}\left(q_{a}\right)$. Similarly $k \sigma(q)$ is the vector with components $k_{a} \sigma_{a}\left(q_{a}\right)$. 
THEOREM 6. Let $G=(N, A)$ be a connected network.

If $p_{*}$ solves the primal problem $(\mathrm{P})$, defined above, then $q=k \sigma\left(E^{\top} p_{*}\right)$ solves the dual problem (D).

If $q_{*}$ solves the dual problem (D), and $q_{*}=k \sigma\left(E^{\top} p\right)$ for some $p$ with $p(0)=0$, then $p$ solves the primal problem $(\mathrm{P})$.

At the solutions, $U\left(q_{*}\right)=-V_{b}\left(p_{*}\right)$.

The constraints $E q=b$ restrict $q$ to lie on a certain hyperplane. For type (H) networks, the minimisation problem, Problem (D), is to find the point $q_{*}$ on this hyperplane which is closest to the origin in a certain $l_{p}$ norm. For networks not of type (H), Problem (D) is to find the point $q_{*}$ on this hyperplane at which $U(q)$ is minimised.

Theorem 2 can be rephrased to give a characterisation of $l_{p}$ norms amongst certain classes of convex functions $U$. Other recent characterisations of $l_{p}$ norms occur in $[6,7]$.

\section{Proof of Theorem 1}

The theorems in this subsection do not depend on the "network" aspects of the optimisation problem. We begin with a very easy general monotonicity result.

LEMMA 7. Consider any continuous function $V \mathbb{R}^{|N|} \times \mathbb{R}^{|A|} \rightarrow \mathbb{R}$, and denote the variables in $\mathbb{R}^{|N|}$ by $p$, and the parameters in $\mathbb{R}^{|A|}$ by $k$. Suppose

$$
k \leq \hat{k} \quad \Rightarrow \quad V(p, k) \leq V(p, \hat{k}) \quad \forall p .
$$

Suppose that, for all $k, p_{*}(k)$ exists satisfying

$$
V\left(p_{*}(k), k\right)=\min \left\{V(p, k) \mid p \in \mathbb{R}^{|N|}\right\} .
$$

Then

$$
k \leq \hat{k} \quad \Rightarrow \quad V\left(p_{*}(k), k\right) \leq V\left(p_{*}(\hat{k}), \hat{k}\right) .
$$

PROOF. Inequality (6.1) gives, with $k \leq \hat{k}$,

$$
V\left(p_{*}(\hat{k}), k\right) \leq V\left(p_{*}(\hat{k}), \hat{k}\right) .
$$

Inequality (6.2) gives

$$
V\left(p_{*}(k), k\right) \leq V\left(p_{*}(\hat{k}), k\right) .
$$


The result follows from these two inequalities.

REMARK. If, as in problem $(P), V_{b}(p, k)$ at a fixed $p$ is concave in $k$, then $V_{b}\left(p_{*}(k), k\right)$ is concave in $k$.

The proofs of both theorems in this section depend on the homogeneity of $V_{0}$. Calculus arguments give that the homogeneity is equivalent to

$$
p^{\top} D V_{0}(p)=(s+1) V_{0}(p) \quad \forall p,
$$

and the calculus condition for a minimum of $V_{b}$ is $D V_{0}\left(p_{*}\right)=b$. In the context of the network problem, the essential consequences of homogeneity are contained in the following lemma, whose proof does not need calculus.

LEMMA 8. For a type $(\mathrm{H})$ network, at the solutions $p_{*}$ and $q_{*}$ of Problems $(\mathrm{P})$ and (D),

$$
P=p_{*}^{\top} b=V_{0}\left(p_{*}\right)-V_{b}\left(p_{*}\right)=(s+1) V_{0}\left(p_{*}\right)=\frac{s+1}{s} U\left(q_{*}\right) .
$$

PROOF. For a type (H) network, substituting the power-law expressions for $R_{a}$ and $S_{a}$ in the formulae for $U$ and $V_{0}$ respectively, and using the fact that for each $\operatorname{arc} a \sim(i, j)$,

$$
\left|q_{* a}\right|=k_{a}\left|p_{*}(i)-p_{*}(j)\right|^{s},
$$

it follows that $U\left(q_{*}\right)=s V_{0}\left(p_{*}\right)$. (See (6.10) and (4.1).) This gives the rightmost equality of (6.1). The duality Theorem 6 stated that $U\left(q_{*}\right)=-V_{b}\left(p_{*}\right)=$ $-V_{0}\left(p_{*}\right)+p_{*}^{\top} b$, or equivalently $p_{*}^{\top} b=V_{0}\left(p_{*}\right)-V_{b}\left(p_{*}\right)=V_{0}\left(p_{*}\right)+U\left(q_{*}\right)$. From this and the equality of the first sentence, (6.3) follows.

PROOF OF THEOREM 1. Lemma 7 applies when $V=V_{b}$ and ensures that

$$
\hat{k} \geq k \quad \Rightarrow \quad V_{b}\left(p_{*}(k), k\right) \leq V_{b}\left(p_{*}(\hat{k}), \hat{k}\right) .
$$

Then (6.4) combined with duality $U\left(q_{*}\right)=-V_{b}\left(p_{*}\right)$, and with (6.3) yields Theorem 1.

With the same hypotheses as in Theorem 1, it is possible to prove more about how the power changes as conductivity factors are changed. This additional result has been separated from Theorem 1 because it is less closely related to the Braess phenomena than is Theorem 1. 
THEOREM 9. Consider flows in a type $(\mathrm{H})$ network with the same network topology, the same $b$, but varying conductivity factors $k$. The power-loss $P$ (i) is a convex function of the $k$, and (ii) is a concave function of the $r$ where $r_{a}=k_{a}^{-1 / s}$.

PROOF. (i) We suppose that the conductivity factors $k$ vary as follows:

$$
k_{\tau}=(1-\tau) k_{0}+\tau k_{1}, \quad \tau \in[0,1] .
$$

Let $p_{\tau}$ be the minimiser of $V_{b, \tau}(p)=V_{0, \tau}(p)-b^{\top} p$. The power-loss $P_{\tau}=b^{\top} p_{\tau}$. In particular

$$
V_{0,0}\left(p_{0}\right)-P_{0} \leq V_{0,0}\left(p_{\tau}\right)-P_{\tau}, \quad V_{0,1}\left(p_{1}\right)-P_{1} \leq V_{0,1}\left(p_{\tau}\right)-P_{\tau} .
$$

Equation (6.3) can be applied to the minimisers $p_{0}$ and $p_{1}$, so that multiplying the two preceding inequalities by $s+1$ gives:

$$
-s P_{0} \leq(s+1)\left(V_{0,0}\left(p_{\tau}\right)-P_{\tau}\right), \quad-s P_{1} \leq(s+1)\left(V_{0,1}\left(p_{\tau}\right)-P_{\tau}\right) .
$$

Taking $1-\tau$ times the first of these plus $\tau$ times the second gives

$$
-s\left((1-\tau) P_{0}+\tau P_{1}\right) \leq(s+1)\left(V_{0, \tau}\left(p_{\tau}\right)-P_{\tau}\right) .
$$

Equation (6.3) can also be applied to the minimiser $p_{\tau}$, so that

$$
P_{\tau}=(s+1) V_{0, \tau}\left(p_{\tau}\right) .
$$

Finally, we eliminate $V_{0, \tau}\left(p_{\tau}\right)$ and then (6.7) and (6.8) give

$$
-s\left((1-\tau) P_{0}+\tau P_{1}\right) \leq-s P_{\tau} .
$$

This establishes the convexity result which we were required to prove.

(ii) Recall that $r$ is the vector of all the $r_{a}$ and $r_{a}=k_{a}^{-1 / s}$. The function $U$ defined at Problem (D) can be written

$$
U(q)=\frac{s}{s+1} \sum_{a \in A} r_{a}\left|q_{a}\right|^{(s+1) / s} .
$$

We now follow steps similar to part (i). Suppose

$$
r_{\tau}=(1-\tau) r_{0}+\tau r_{1}, \quad \tau \in[0,1] .
$$

Let $q_{\tau}$ be the minimisers of $U_{\tau}$ over the set of $q$ such that $E q=b$. Let $P_{\tau}$ be the corresponding powers. Then

$$
U_{0}\left(q_{\tau}\right) \geq U_{0}\left(q_{0}\right)=\frac{s}{s+1} P_{0}, \quad U_{1}\left(q_{r}\right) \geq U_{1}\left(q_{1}\right)=\frac{s}{s+1} P_{1} .
$$


Multiplying the first by $(1-\tau)$ and the second by $\tau$ and adding gives

$$
P_{\tau} \geq(1-\tau) P_{0}+\tau P_{0} .
$$

This establishes the required concavity of the power in $r$.

An alternative argument using both parts (i) and (ii) to give the result of Theorem 1 follows. Let $a$ be fixed. Let the power as a function of $k_{a}$ be denoted $P k\left(k_{a}\right)$. Let the power as a function of $r_{a}=k_{a}^{-1 / s}$ be denoted $\operatorname{Pr}\left(r_{a}\right)$. Omit the subscript $a$ in the rest of this proof. Consider $k_{0}<k_{1}$. Since $P k$ is convex in $k$ and $P r$ is concave in $k^{-1 / s}$,

$$
\frac{d P k}{d k}\left(k_{1}\right)-\frac{d P k}{d k}\left(k_{0}\right) \geq 0, \quad \frac{d P r}{d r}\left(k_{1}^{-1 / s}\right)-\frac{d P r}{d r}\left(k_{0}^{-1 / s}\right) \geq 0 .
$$

Now

$$
\frac{d P k}{d k}(k)=-\frac{1}{s} k^{-(s+1) / s} \frac{d P r}{d r}\left(k^{-1 / s}\right) .
$$

Combining the immediately preceding displayed items gives

$$
\left(k_{0}^{(s+1) / s}-k_{1}^{(s+1) / s}\right) \frac{d P k}{d k}\left(k_{0}\right) \geq 0, \quad \text { hence } \frac{d P k}{d k}\left(k_{0}\right) \leq 0,
$$

as required.

\section{Proof of Theorem 2}

In contrast to Section 6, all the proofs in Sections 7 and 8 depend on the network structure. In this section, we complete the proof of Theorem 2 by proving Lemma 3.

The next lemma says that if two arcs with the same conductivity function $\sigma$ (up to a multiplicative constant) placed in series give the same function $\sigma$ (up to a multiplicative constant-which, we remark, is a very strong requirement), then $\sigma$ is a power law, $\sigma(t)=\sigma(1) t^{s}$ for $t \geq 0$. The lemma is effectively a uniqueness statement for a certain functional equation.

LEMMA 10. Let $\sigma(0, \infty) \rightarrow(0, \infty)$ be nondecreasing and absolutely continuous on compact intervals. Suppose for all $h>0$ there exists $\theta(h)>0$ such that for all $v>0$, there is a $t \in(0, v)$ such that

$$
\theta(h) \sigma(v)=h \sigma(t)=\sigma(v-t) .
$$


Then, there exists $s>0$ such that $\sigma(t)=\sigma(1) t^{s}$.

PROOF. Taking $h=1$, there is a $\theta(1)$ such that, for all $v$, there is a $t$ with

$$
\theta(1) \sigma(v)=\sigma(t)=\sigma(v-t) .
$$

Since $\sigma$ is nondecreasing for all $v>0$,

$$
\sigma(v / 2)=\theta(1) \sigma(v)
$$

Hence for $n \in \mathbb{N}$,

$$
\sigma\left(v / 2^{n}\right)=\theta(1)^{n} \sigma(v) .
$$

Take $h=1 / \theta(1)$. For all $v$, there is a $t$ with

$$
\theta\left(\theta(1)^{-1}\right) \sigma(v)=\theta(1)^{-1} \sigma(t)=\sigma(v-t) .
$$

The central term is $\sigma(2 t)$ by (7.1). If $t \leq v / 3$, we have $2 t \leq 2 v / 3 \leq v-t$, giving $\theta(h) \sigma(v)=\sigma(2 v / 3)$, since $\sigma$ is nondecreasing. If $t>v / 3$, we have $v-t \leq 2 v / 3 \leq 2 t$, and again $\theta(h) \sigma(v)=\sigma(2 v / 3)$. Using (7.1), this gives

$$
\sigma(v / 3)=\theta(1) \theta\left(\theta(1)^{-1}\right) \sigma(v) .
$$

Continuing, for all $m, n \in \mathbb{N}$, there is a $\lambda(m, n)>0$ such that, for all $v>0$,

$$
\sigma\left(m v / 2^{n}\right)=\lambda(m, n) \sigma(v) .
$$

Hence, unless $\sigma$ is identically zero, $\sigma(v)>0$ for all $v>0$. Letting $v=\exp \xi$, for $\xi \in \mathbb{R}$, and assuming the left-hand side is positive so we can take logarithms,

$$
\log \left(\sigma\left(\exp \left(\xi+\log \left(m / 2^{n}\right)\right)\right)\right)=\log (\lambda(m, n))+\log (\sigma(\exp \xi)) .
$$

Dividing by $\log \left(m / 2^{n}\right)$ and letting $\left(m / 2^{n}\right)$ tend to one, we have, for $\xi$ where $(\log \circ \sigma \circ \exp )^{\prime}(\xi)$ exists, that it is a positive constant independent of $\xi$ (as the $m, n$ are independent of $\xi$ ).

Thus there are $s \geq 0$ and $c \in \mathbb{R}$, with

$$
\log \circ \sigma \circ \exp (\xi)=s \xi+c \forall \xi, \quad \text { or } \quad \sigma(v)=\exp (c) v^{s} \forall v .
$$

The next two theorems from earlier papers, and our discussion in Section 3, give some indications towards our proof of Theorem 2 . We begin with definitions from Riordan and Shannon [19]. 
DEFINITION. Let $n_{0}$ and $n_{f}$ be given nodes of a network $G$. The network $G$ is said to be series-parallel with respect to $n_{0}$ and $n_{f}$ if through each arc of $G$ there is at least one path from $n_{0}$ to $n_{f}$ not touching any node twice, and no two of these paths pass through any arc in opposite directions.

An equivalent inductive definition is as follows. The one-arc graph $G_{0}$ with $A_{0}=\left(n_{0}, n_{f}\right)$ is defined to be series-parallel. A network is series-parallel with respect to $n_{0}$ and $n_{f}$ if it is either (i) a connection of a series-parallel network with respect to $n_{0}$ and $n_{i}$ in series with a second series-parallel network with respect to $n_{i}$ and $n_{f}$, or (ii) a parallel connection of two networks both series-parallel with respect to $n_{0}$ and $n_{f}$.

(The Wheatstone bridge graph of the example in Section 3 is not seriesparallel with respect to $\{0,3\}$, but is series-parallel with respect to $\{1,2\}$.) $A$ two-terminal network is said to be series-parallel if it is series-parallel with respect to the two terminal nodes.

THEOREM 11. For series-parallel two-terminal networks, the power-loss $P$ decreases when any conductivity factor $k$ increases.

Characterisations of series-parallel networks are also known. See Duffin [13].

THEOREM 12. A two terminal network is series-parallel if and only if there is no embedded network having the Wheatstone bridge configuration.

We now return to the proof of Lemma 3, knowing that Wheatstone bridges must be used as test networks.

LEMMA 13. Consider the Wheatstone bridge graph with nodes numbered as in (3.1). The arcs in $A$ are indexed by the numbers 1 to 5 in the order listed in (3.1). Suppose the conductivity functions satisfy Assumption $A(\sigma)$.

Let $M(k, b)$ be the statement: for the given value of $b$ and for all positive values of $k_{1}, k_{2}, k_{4}, k_{5}$ and nonnegative values of $k_{3}$, the power-loss is nonincreasing as the conductivity factor $k_{3}$ of the arc $3=(1,2)$ increases.

Suppose there exists a nonzero $b$ with $b(1)=0=b(2)$, such that $M(k, b)$ holds. Then there exists $\lambda$ such that

$$
\frac{\sigma_{1}^{\prime}(x)}{\sigma_{1}(x)} \frac{\sigma_{2}(x)}{\sigma_{2}^{\prime}(x)}=\lambda \forall x>0, \quad \frac{\sigma_{4}^{\prime}(y)}{\sigma_{4}(y)} \frac{\sigma_{5}(y)}{\sigma_{5}^{\prime}(y)}=\lambda \forall y>0,
$$

and there exist numbers $\mu_{1,2}, \mu_{4,5}$ such that

$$
\sigma_{1}(x)=\exp \left(\mu_{1,2}\right)\left(\sigma_{2}(x)\right)^{\lambda} \forall x>0, \quad \sigma_{4}(y)=\exp \left(\mu_{4,5}\right)\left(\sigma_{5}(y)\right)^{\lambda} \forall y>0 .
$$


REMARK. (i) If there exists a nonzero $b$ such that $M(k, b)$ holds, then for all nonzero $b, M(k, b)$ holds. To see this, consider scaling both $b$ and $k$ by the same positive factor.

(ii) The lemma remains true when $M(k, b)$ is replaced by $M_{0}(k, b)$ involving $k_{3}$ increasing from zero, rather than any initial nonnegative value of $k_{3}$.

PROOF. The flow function on $\operatorname{arc} j$ is $k_{j} \sigma_{j}, j=1, \ldots, 5$. Take $p(0)=0$, let $p(i)$ denote the head at node $i$, let $p=(p(1), p(2), p(3))^{\top}$ and let $k=$ $\left(k_{1}, k_{2}, k_{3}, k_{4}, k_{5}\right)$. Define $F \mathbb{R}^{3} \oplus \mathbb{R}^{5} \rightarrow \mathbb{R}^{3}$ by

$$
\begin{aligned}
& F_{1}(p, k)=k_{1} \sigma_{1}(p(1))+k_{3} \sigma_{3}(p(1)-p(2))+k_{4} \sigma_{4}(p(1)-p(3)), \\
& F_{2}(p, k)=k_{2} \sigma_{2}(p(2))+k_{3} \sigma_{3}(p(2)-p(1))+k_{5} \sigma_{5}(p(2)-p(3)), \\
& F_{3}(p, k)=k_{4} \sigma_{4}(p(3)-p(1))+k_{5} \sigma_{5}(p(3)-p(2)) .
\end{aligned}
$$

At fixed $k, k_{j}>0$ for $j \neq 3, k_{3} \geq 0, F=D V_{0}$ and, by Duffin's Existence Theorem (stated with Theorem 4 ), there is a unique $p$ satisfying the network equilibrium problem,

$$
F(p, k)=(0,0, b(3)) .
$$

Consider the Jacobian $D_{p} F(p, k)$, where $D_{p}$ denotes the derivative with respect to $p$ with $k$ held constant. We have

$$
D_{p} F(p, k)=\left(\begin{array}{ccc}
k_{1} \sigma_{1}^{\prime}+k_{3} \sigma_{3}^{\prime}+k_{4} \sigma_{4}^{\prime} & -k_{3} \sigma_{3}^{\prime} & -k_{4} \sigma_{4}^{\prime} \\
-k_{3} \sigma_{3}^{\prime} & k_{2} \sigma_{2}^{\prime}+k_{3} \sigma_{3}^{\prime}+k_{5} \sigma_{5}^{\prime} & -k_{5} \sigma_{5}^{\prime} \\
-k_{4} \sigma_{4}^{\prime} & -k_{5} \sigma_{5}^{\prime} & k_{4} \sigma_{4}^{\prime}+k_{5} \sigma_{5}^{\prime}
\end{array}\right),
$$

where, for $a \sim(i, k), \sigma_{a}^{\prime} \equiv \sigma_{a}^{\prime}(p(i)-p(k))$. (Recall that $\sigma_{a}^{\prime}(-t)=\sigma_{a}^{\prime}(t)$.) Either directly from this, or indirectly from the convexity of $V_{0}$, we have $\delta=$ $\operatorname{det}\left(D_{p} F(p, k)\right)>0$. The Cramer's-rule formula for the inverse of the matrix $D_{p} F(p, k)$ is also useful. It is

$$
\delta\left(D_{p} F(p, k)\right)^{-1}=\left(\begin{array}{rrr}
m(2,3 ; 2,3) & -m(1,3 ; 2,3) & m(1,2 ; 2,3) \\
-m(1,3 ; 2,3) & m(1,3 ; 1,3) & -m(1,2 ; 1,3) \\
m(1,2 ; 2,3) & -m(1,2 ; 1,3) & m(1,2 ; 1,2)
\end{array}\right)
$$

where

$$
m(i, j ; k, l)=\frac{\partial\left(F_{i}, F_{j}\right)}{\partial(p(k), p(l))} .
$$

Since the smoothness hypotheses of the Implicit Function Theorem hold (provided $p(1) \neq p(2)$, since then all the $q_{a}$ will be nonzero so that $\sigma_{a}$ is $C^{1}$ there), 
(7.7) gives $p$ as a $C^{1}$ function of $k$ with

$$
\frac{\partial p(3)}{\partial k_{3}}=-(0,0,1)\left(D_{p} F(p, k)\right)^{-1} \frac{\partial F}{\partial k_{3}} .
$$

Now

$$
\frac{\partial F}{\partial k_{3}}=\sigma_{3}(p(1)-p(2))(1,-1,0)^{\top},
$$

from which

$$
\frac{\partial p(3)}{\partial k_{3}}=-\left(\operatorname{det}\left(D_{p} F(p, k)\right)\right)^{-1} \sigma_{3}(p(1)-p(2)) M,
$$

where

$$
\begin{aligned}
M & =-(0,0,1) \delta\left(D_{p} F(p, k)\right)^{-1}(1,-1,0)^{\top}, \\
& =m(1,2 ; 2,3)+m(1,2 ; 2,3), \\
& =k_{2} \sigma_{2}^{\prime}(p(2)) k_{4} \sigma_{4}^{\prime}(p(3)-p(1))-k_{1} \sigma_{1}^{\prime}(p(1)) k_{5} \sigma_{5}^{\prime}(p(3)-p(2)) .
\end{aligned}
$$

Now $P=b(3) p(3)$. If $p(3)$ is to be monotonic in $k_{3}, M$ must change sign at points where $\sigma_{3}(p(1)-p(2))$ changes sign. Hence $p(1)=p(2)$ implies

$$
k_{2} \sigma_{2}^{\prime}(p(2)) k_{4} \sigma_{4}^{\prime}(p(3)-p(1))=k_{1} \sigma_{2}^{\prime}(p(1)) k_{5} \sigma_{5}^{\prime}(p(3)-p(2)) .
$$

Since $F_{1}(p, k)=0$ and $F_{2}(p, k)=0, p(1)=p(2)$ also implies

$$
\begin{aligned}
& k_{1} \sigma_{1}(p(1))=k_{4} \sigma_{4}(p(3)-p(1)), \\
& k_{2} \sigma_{2}(p(2))=k_{5} \sigma_{5}(p(3)-p(2)) .
\end{aligned}
$$

With $b(3)>0$, given $x, y>0$, there are positive values of $k_{1}, k_{2}, k_{4}, k_{5}$ such that $p(1)=x=p(2)$ and $p(3)=x+y$. (We just take $k_{1}$ so that $k_{1} \sigma_{1}(x)<b(3)$ and define $k_{2}$ by

$$
k_{1} \sigma_{1}(x)+k_{2} \sigma_{2}(x)=b(3),
$$

and $k_{4}$ and $k_{5}$ are given by (7.10) and (7.11).) Thus, (7.9), (7.10) and (7.11) give

$$
\frac{\sigma_{1}^{\prime}(x)}{\sigma_{1}(x)} \frac{\sigma_{5}^{\prime}(y)}{\sigma_{5}(y)}=\frac{\sigma_{2}^{\prime}(x)}{\sigma_{2}(x)} \frac{\sigma_{4}^{\prime}(y)}{\sigma_{4}(y)} .
$$

From this, with $\Lambda_{j}=\log \sigma_{j}$, there exists $\lambda \in \mathbb{R}$ such that

$$
\frac{\Lambda_{1}^{\prime}(x)}{\Lambda_{2}^{\prime}(x)}=\lambda \quad \forall x>0, \quad \frac{\Lambda_{4}^{\prime}(y)}{\Lambda_{5}^{\prime}(y)}=\lambda \quad \forall y>0 .
$$


Rewriting the second of these gives

$$
\Lambda_{4}^{\prime}(y)=\lambda \Lambda_{5}^{\prime}(y) \quad \forall y>0,
$$

giving

$$
\Lambda_{4}(y)=\lambda \Lambda_{5}(y)+\mu \quad \forall y>0,
$$

and

$$
\sigma_{4}(y)=\exp (\mu)\left(\sigma_{5}(y)\right)^{\lambda} .
$$

The result with arcs 1 and 2 is proved similarly, and the proof of the lemma is then complete.

PROOF OF LEMMA 3. The proof is in two parts, the first of which applies with $v \geq 5$.

(i) Let $\left(N_{1}, A_{1}\right)$ be as in (3.1) with arcs indexed as in Lemma 13, and let $\varphi A_{1} \rightarrow \mathbb{N}_{v}$ satisfy $\varphi$ on $\operatorname{arc}(0,2)$ is 2 , and $\varphi$ on $\operatorname{arc}(0,1)$ is $j \neq 2$. By Lemma 13 , there are positive $\lambda_{j}$ and $\mu_{j}$ such that for all $x \in \mathbb{R}, \sigma_{j}(x)=\mu_{j} \sigma_{2}(x)^{\lambda_{j}}$. By (7.6), since

$$
\frac{\sigma_{j}^{\prime}(x)}{\sigma_{j}(x)}=\lambda_{j} \frac{\sigma_{2}^{\prime}(x)}{\sigma_{2}(x)}
$$

if $l, m, n$ and 2 are four elements of $\mathbb{N}_{v}$, then, with $\lambda_{2}$ defined to be 1 ,

$$
\lambda_{2} \lambda_{m}=\lambda_{l} \lambda_{n}
$$

Equation (7.13) is true for all permutations of the different integers $l, m, n$. From this and $\lambda_{2}=1$ it follows that $\lambda_{j}=1$ for all $j$. Writing $\sigma$ for $\sigma_{2}$, for all $j \in \mathbb{N}_{v}$ there is $\mu_{j}$ with $\sigma_{j}=\mu_{j} \sigma$.

(ii) We now move to $v \geq 6$. Define

$$
\begin{aligned}
& N_{2}=\{0,1,2,3,4\}, \\
& A_{2}=\{(0,4),(4,1),(0,2),(1,2),(1,3),(2,3)\} .
\end{aligned}
$$

(We have inserted the node 4 into the $\operatorname{arc}(0,1)$. The two $\operatorname{arcs} a_{1}=(0,4)$, $a_{2}=(4,1)$ are in series. The set is essentially a Wheatstone bridge network, with the pair not on the central arc $(1,2)$.) We shall regard this as a twoterminal network with the selected terminals 0 and 3 , and $b(4)=0$. (This is different than conventions elsewhere in this paper and in [15]: it is only used in the proof.) Rather than repeating all steps of an analysis like Lemma 13 on 
$\left(N_{2}, A_{2}\right)$, we apply Lemma 13 to the Wheatstone bridge $\left(N_{1}, A_{1}\right)$ with $(0,1)$ having conductivity function $\tilde{\sigma}$ with

$$
\tilde{\sigma}^{-1}(q)=\sigma^{-1}(q / h)+\sigma^{-1}(q),
$$

where $h>0$ is given. That is, $\tilde{\sigma}^{-1}$ has as its graph the characteristic curve of the two-terminal network $\left(N_{3}, A_{3}\right)$ where $N_{3}=\{0,4,1\}$ and $A_{3}=\{(0,4),(4,1)\}$, $b(4)=0$, with flow functions $h \sigma$ on $(0,4)$ and $\sigma$ on $(4,1)$. In both the networks $\left(N_{1}, A_{1}\right)$ and $\left(N_{2}, A_{2}\right), b(i)=0$ except when $i=0$ and $i=3$. We will have the same relationship between heads, flows, and $b$ in $\left(N_{1}, A_{1}\right)$, with flow functions $k_{1} \tilde{\sigma}$ on $(0,1), k_{2} \sigma$ on $(0,2), k_{3} \sigma$ on $(1,2), k_{4} \sigma$ on $(1,3), k_{5} \sigma$ on $(2,3)$, as in $\left(N_{2}, A_{2}\right)$, with flow functions $k_{1} h \sigma$ on $(0,4), k_{1} \sigma$ on $(4,1)$, and exactly the same as in $\left(N_{1}, A_{1}\right)$ on the other four arcs.

We use, again, the fact that the power is nonincreasing in $K_{a}$. Applying Lemma 13 to $\left(N_{1}, A_{1}\right)$, there is $\theta(h)>0$ and $\lambda>0$ such that for $x \in \mathbb{R}$,

$$
\tilde{\sigma}(x)=\theta(h) \sigma(x)^{\lambda},
$$

and, by (7.13), $\lambda=1$.

We now apply Lemma 10 to $\left(N_{3}, A_{3}\right)$ with flow functions $h \sigma$ on $(0,4)$ and $\sigma$ on $(4,1)$. Given potentials $p(0)=0$ and $p(1)$, the flow $q$ is given by $q=\theta(h) \sigma(p(1))$. There exists a potential $p(4)$ such that on $(0,4)$ we have $q=h \sigma(p(4))$ and on $(4,1)$ we have $q=\sigma(p(1)-p(4))$. Hence there is an $s>0$ such that, for all $t \geq 0, \sigma(t)=\sigma(1) t^{s}$.

Hence, for all $a \in \mathbb{N}_{\nu}, \sigma_{a}(t)=\sigma_{a}(1) t^{s}$. This completes the proof.

REMARK. Work is in progress on the following variation on Theorem 2. Rather than fixing conductivity functions $\sigma_{a}$ and varying conductivity factors $k_{a}$, it may be appropriate to fix resistance functions $\rho_{a}$ and vary resistance factors $r_{a}$. These can be defined by changing the position of the scalar factor in (2.2) to give $q_{a}=\sigma_{a}\left((p(i)-p(k)) / r_{a}\right)$. In the context of the pipe-network problem, changes in $r_{a}$ come from varying the lengths of the pipes.

The analogue of Lemma 10 is as follows. If two arcs with the same resistance function $\rho$ (up to a multiplicative constant) placed in parallel give the same function $\rho$ (up to a multiplicative constant) then $\rho$ is a power law, $\rho(t)=\rho(1) t^{1 / s}$ for $t \geq 0$.

An informal way to consider possible variations on Theorem 2 and Lemma 3 is as follows. Suppose that one is given $v(\geq 6)$ very large rolls of wire, with each roll being identifiable by its colour, say. The rolls of wire are made of 
rather exotic electrical conductors. For each type of wire $w$, the form of the resistance function $\rho_{w}$ is constant along the wire in roll $w$. By cutting a length $l$ of wire from roll $w$, one obtains an element with resistance $r(l) \rho_{w}$ (with larger $r(l)$ corresponding to longer $l$ ). Electrical networks can be built making their arcs from lengths $l_{w}$ of wire from roll $w$ assembled in various kinds of networks. If, no matter which electrical network is built, the power-loss $P$ increases whenr is increased, we expect that the network would have to be of type (H). See the authors' 1992 University of Auckland research report for results.

\section{Further results}

The following theorems (i) show, for the Wheatstone graph, that type (H) is not necessary to have power-loss decreasing in $k_{a}$, and (ii) show that $v \geq 6$ is necessary in Lemma 3.

THEOREM 14. Suppose, in addition to the hypotheses of Lemma 13, that the conductivity functions on each arc are the same, that is, $k_{a} \sigma_{a}=k_{a} \sigma$ for some $\sigma$ satisfying Assumption $A(\sigma)$. If it is given that at any $k \in \mathbb{R}^{5}, k \geq 0$ satisfying Assumption $A(k)$, the power-loss is a decreasing function of any $k_{a}$ then $\log \sigma$ is concave on $(0, \infty)$.

Proof. Given $0<p(2)<p(1)$, we let $p(3)=p(1)+p(2)$, and choose $k_{1}$, $k_{2}, k_{4}, k_{5}$ positive so that (7.10) and (7.11) hold, and $k_{3}=0$. Let $0 \leq b(3)=$ $F_{3}(p, k)$. Since $\partial p(3) / \partial k_{3} \leq 0$ at these values of $p$ and $k,(7.8)$ gives $M \geq 0$, which gives $\left(\Lambda^{\prime}(p(2))\right)^{2} \geq\left(\Lambda^{\prime}(p(1))\right)^{2}$ where $\Lambda=\log \sigma$, and hence $\log \sigma$ is concave on $(0, \infty)$.

THEOREM 15. Consider the Wheatstone bridge graph, and two-terminal flows with $b(1)=0=b(2)$. Suppose that the conductivity functions on each arc are the same, that is $k_{a} \sigma_{a}=k_{a} \sigma$ for some $\sigma$ satisfying Assumption $A(\sigma)$. The power-loss does not increase if $k_{a}$ is increased

(i) with no further restrictions on $\sigma$, if $a$ is any arc except $(1,2)$,

(ii) if $a$ is $(1,2)$ provided $\log \sigma$ is concave.

Proof. Suppose $b(3)>0$. There are two cases to consider: when $a$ is the central arc $3=(1,2)$ and when it is one of the other arcs. 
(i) We begin with the case that $a$ is not the central arc. To show $p(3)$ is decreasing in $k_{j}, j \neq 3$, by symmetry it is enough to let $j=1$, that is, $a=(0,1)$. Since

$$
\frac{\partial p(3)}{\partial k_{1}}=-(0,0,1)\left(D_{p} F(p, k)\right)^{-1} \frac{\partial F}{\partial k_{1}},
$$

and

$$
\frac{\partial F}{\partial k_{1}}=\sigma(p(1))(1,0,0)^{\top},
$$

then

$$
\frac{\partial p(3)}{\partial k_{1}}=-\sigma(p(1))\left(\operatorname{det}\left(D_{p} F(p, k)\right)\right)^{-1} M_{1},
$$

where

$$
M_{1}=m(1,2 ; 2,3)=\operatorname{det}\left(\frac{\partial\left(F_{1}, F_{2}\right)}{\partial(p(2), p(3))}\right) .
$$

The fact that $\operatorname{det}\left(D_{p} F(p, k)\right)>0$ shows that the proof depends on establishing the sign of $M_{1}$. Expanding the determinant defining $M_{1}$ gives

$$
M_{1}=k_{3} \sigma^{\prime}(p(1)-p(2)) k_{5} \sigma^{\prime}(p(2)-p(3))+k_{4} \sigma^{\prime}(p(1)-p(3)) \frac{\partial F_{2}}{\partial p(2)},
$$

where

$$
\frac{\partial F_{2}}{\partial p(2)}=k_{2} \sigma^{\prime}(p(2))+k_{3} \sigma^{\prime}(p(2)-p(1))+k_{5} \sigma^{\prime}(p(2)-p(3)) .
$$

Since $\sigma^{\prime}>0$ this establishes the sign of $M_{1}$ and hence shows that $p(3)$ is a decreasing function of $k_{1}$.

(ii) Next consider the case $a=(1,2)$, the central arc. By (7.8), to show $p(3)$ is decreasing in $k_{3}$, we need to show $M \geq 0$ for $p(1)>p(2)$, and $M \leq 0$ for $p(1)<p(2)$. Here $M$ is the expression occurring in (7.8). Write $\Lambda=\log \sigma$ and eliminate the $\sigma^{\prime}$ terms from $M$ using $\sigma^{\prime}=\sigma \Lambda^{\prime}$.

First consider $p(1)>p(2)$. As we have also supposed $b(3)>0$, we have $p(3)>p(1)>p(2)>p(0)=0$. We have

$$
M=k_{1} k_{2} \sigma(p(1)) \sigma(p(2))\left(L_{1} K_{1}+L_{2} K_{2}\right)
$$

where

$$
L_{1}=\left(\Lambda^{\prime}(p(3)-p(1)) \Lambda^{\prime}(p(2))-\Lambda^{\prime}(p(1)) \Lambda^{\prime}(p(3)-p(2))\right),
$$




$$
\begin{aligned}
L_{2} & =\left(\Lambda^{\prime}(p(1)) \Lambda^{\prime}(p(3)-p(2))\right), \\
K_{1} & =\left(1+\frac{k_{3} \sigma(p(1)-p(2))}{k_{1} \sigma(p(1))}\right), \\
K_{2} & =\left(\frac{k_{3} \sigma(p(1)-p(2))}{k_{1} \sigma(p(1))}+\frac{k_{3} \sigma(p(1)-p(2))}{k_{2} \sigma(p(2))}\right) .
\end{aligned}
$$

Since $\Lambda^{\prime}$ is decreasing, with $p(1)>p(2)$ and hence $p(3)-p(2)>p(3)-p(1)$, one can deduce that $L_{1} \geq 0$. Since $p(3)>p(1)>p(2)>p(0)=0$, both $K_{1}$ and $K_{2}$ are nonnegative. Since $\sigma^{\prime} \geq 0, L_{2} \geq 0$. This establishes that $M \geq 0$ as required.

A similar argument, involving a different reorganisation of the terms of $M$, can be used to show $M \leq 0$ if $p(1)<p(2)$.

\section{Acknowledgement}

G. Keady acknowledges the support of a Research Fellowship awarded by the U.G.C. of New Zealand. The work in this paper is a by-product of a symbolic-numeric computing case study, involving a computational package from the University of Waikato. The optimisation problem (P) did not pose severe algebra demands, but was, for moderately large networks, beyond the usual scope for numerics of the widely used computer-algebra packages, without some argumentation. See Keady [15] or Broughan et al. [5, 4] for further details.

The authors thank colleagues at Auckland and Waikato: Kim Prisk and Murray Jorgensen for calling their attention to the authors of reference [8], and Les Foulds for providing the bibliographic details of references $[2,14,18]$.

\section{References}

[1] D. P. Bertsekas, P. A. Hosein and P. Tseng, "Relaxation methods for network flow problems with convex arc costs", SIAM J. Control Optim. 25 (1987) 1219-1243.

[2] D. Braess, "Über ein Paradoxen aus der Verkehrsplanung", Unternehmensforschung 12 (1968) 256-268.

[3] C. A. Brebbia and A. J. Ferrante, Computational hydraulics (Butterworth, London, 1983).

[4] K. A. Broughan and G. Keady, "Numlink and Naglink: links to the NAG library from SENAC and Macsyma", in Proceedings of the Workshop on Symbolic and Numeric Com- 
putation (ed. H. Apiola), (Research Reports series of Computing Centre of Helsinki University, 1991) 19-34.

[5] K. A. Broughan, G. Keady, T. D. Robb, M. G. Richardson and M. C. Dewar, "Some symbolic computing links to the NAG numeric library", SIGSAM Bull. 25 (3) (1991) 28-37.

[6] B. Calvert, "When $T$-accretive implies accretive", Houston J. Math. 14 (1988) 1-9.

[7] B. Calvert and S. Fitzpatrick, "Nonexpansive projections onto two-dimensional subspaces of Banach spaces", Bull. Austral. Math. Soc. 37 (1988) 149-160.

[8] J. E. Cohen and P. Horowitz, "Paradoxical behaviour of mechanical and electrical networks", Nature 352 (1991) 699-701.

[9] M. Collins, R. Cooper, J. Helgason, J. Kennington and L. LeBlanc, "Solving the pipe network analysis problem using optimization techniques", Mgmt. Sci. 24 (1978) 747-760.

[10] R. S. Dembo, J. M. Mulvey and S. A. Zenios, "Large scale nonlinear network models and their application”, Oper. Res. 37 (1989) 353-372.

[11] R. J. Duffin, "Nonlinear networks IIa", Bull. Amer. Math. Soc. 53 (1947) 963-971.

[12] R. J. Duffin, "Nonlinear networks IIb", Bull. Amer. Math. Soc 54 (1948) 119-127.

[13] R. J. Duffin, "Topology of series-parallel networks", J. Math. Anal. Appl. 10 (1965) 303318.

[14] C. Fisk, "More paradoxes in the equilibrium assignment problem", Transportation Res. 13B (1979) 305-309.

[15] G. Keady, "SENAC and other symbolic front ends for subsequent numeric computation", Mathematics Research Reports Parts I-IV, University of Waikato, 1991.

[16] L. Leblanc, "An algorithm for the discrete network design problem", Transportation Sci. 9 (1975) 183-199.

[17] H. M. Melvin, “On concavity of resistance functions”, J. Appl. Phys. 27 (1956) 658-659.

[18] J. D. Murchland, "Braess's paradox of traffic flow", Transportation Res. 4 (1970) 391-394.

[19] J. Riordan and C. E. Shannon, "The number of two-terminal series-parallel networks", J. Math. and Phys. 21 (1942) 83-93.

[20] R. T. Rockafellar, Conjugate duality and optimization (SIAM, Philadelphia, 1974).

[21] R. T. Rockafellar, Network flows and monotropic optimisation (Wiley, New York, 1984).

[22] C. E. Shannon and D. W. Hagelbarger, "Concavity of resistance functions", J. Applied Physics 27 (1956) 42-43.

[23] P. Toint, "La modélisation du transport", La Recherche 235 (1991) 1026-1038. 